\title{
Les femmes dans les campagnes publiques de prévention du VIH/sida (1987-1999) : une cible en trompe-l'œil ?
}

\author{
Geneviève PAICHELER ${ }^{1}$ et Audrey SITBON ${ }^{2}$
}

\section{Résumé}

A un moment où l'accélération des nouvelles infections à VIH des femmes, sensible dans tous les pays et particulièrement dramatique dans l'Afrique sub-saharienne, démontre de façon éclatante la dissymétrie des deux sexes devant la maladie et la grande vulnérabilité des femmes, tant physiologique que sociale, il importe de faire retour sur la façon dont la communication préventive s'est adressée à elles dans notre pays. Elles ont été au départ considérées comme des cibles secondaires - la transmission sexuelle de la maladie les concernait moins que les hommes et elles n'avaient pas l'initiative du préservatif - ou comme intermédiaires, du fait du rôle habituel des femmes dans la prévention de leur entourage familial. En revanche, elles étaient mises en avant, Iorsque la communication se centrait sur la solidarité envers les personnes atteintes. On balançait ainsi entre un rôle traditionnel, celui des femmes qui protègent la santé, et un rôle plus innovateur mais irréaliste, celui des femmes qui ont l'initiative dans la sexualité. Ce n'est que très progressivement que la communication a traité de la spécificité des femmes dans la sexualité, que la vulnérabilité des femmes a été reconnue, abordée, et surtout, que les femmes les plus exposées au risque sexuel, originaires des Antilles ou d'Afrique sub-saharienne, ont été la cible de communications. Dans le même temps, la communication ciblée ou hors médias a pris de l'importance par rapport à la communication grand public. Notre article retrace cette évolution entre 1987, date de la première campagne, et 2000 , dans un contexte où les moyens de prévention destinés aux femmes sont soit marginaux, comme le fémidon (préservatif féminin), soit inexistants, comme les microbicides, et ce pour un futur qui paraît indéterminé et certainement long.

Ce n'est que plusieurs années après la découverte des premiers cas de sida, en 1981, que les autorités publiques se sont engagées dans une prévention à grande échelle de cette maladie. Auparavant, par une sorte de contrat tacite entre gouvernants et associations, la question n'était pas abordée sur la scène publique, dans la crainte de provoquer stigmatisation et rejet des personnes vivant avec le VIH. En 1987, la première campagne publique, dont la signature était « le sida ne passera pas par moi ”,

CNRS, UMR 8169, CERMES, Villejuit.

EHESS, CNRS, UMR 8169, CERMES, Villejuif a marqué les esprits, même si le message de prévention qu'elle diffusait était très flou. Tenir compte de la dimension hétérosexuelle de la maladie, en communiquant vers le grand public aurait pu amener à communiquer de manière équilibrée vers les hommes et les femmes, en posant leurs problèmes spécifiques dans la protection de leur sexualité et en abordant les disparités de genre. Nous voulons démontrer ici que cela n'a pas été le cas, notamment pour les femmes. L'objet qui était, et est encore, au centre des recommandations, le préservatif, est utilisé en première instance par les hommes. Et, si telle est leur volonté, ils ont la maîtrise de cette utilisation : ils peuvent soit l'imposer, soit le refuser. Le préservatif vient donc renforcer un déséquilibre de pouvoir entre hommes et femmes sur la sexualité, qui dépasse largement la question de la prévention des infections sexuellement transmissibles, voire le seul cadre de la sexualité (voir l'article de Nathalie Bajos, page 24) [1].

Le fait que les femmes sont longtemps restées des cibles secondaires de la communication était renforcé par les démarches épidémiologiques, qui en faisait également des cibles secondaires de la maladie. Pourtant les données ont fait apparaître précocement que l'infection se diffusait à un rythme soutenu chez les femmes. Mais pas chez n'importe quelles femmes: l'épidémie se concentrait progressivement chez les immigrées, notamment les femmes originaires d'Afrique. Et il a fallu beaucoup de temps pour que ces cibles de l'épidémie deviennent des cibles de la communication. On ne savait pas trop comment s'adresser à cette population et on manquait de données pour comprendre la situation des femmes immigrées atteintes. Bien que sollicitées, les recherches étaient très rares, et ce n'est qu'au tournant des années 2000 que ces femmes ont reçu à la radio et hors des médias des messages traitant directement de leurs problèmes face à la transmission et au vécu de la maladie. Deux raisons pour lesquelles ces actions ne seront pas analysées ici : cette période sort de notre cadre chronologique et nous ne prenons en compte ici que la communication de masse, transitant par des canaux tels que la télévision, la radio, la grande presse et l'affichage, tout en restant centrées sur la France métropolitaine.

Les limites fixées à ce travail tiennent bien sûr à nos recherches antérieures [2,3], ainsi qu'aux exigences que nous nous sommes 
fixées pour les accomplir. En effet, nous n'avons pas pour objectif de faire une sémiologie de la communication en restant centrées sur le contenu du message. Nous sommes plutôt intéressées aux processus politiques qui caractérisent la mise en place de la communication publique, principalement aux interactions et aux controverses entre les différents acteurs en présence : personnel politique, administratifs, chercheurs, membres des associations de lutte contre le sida, des agences de communication. Dans cet objectif, nous avons recueilli et analysé 94 entretiens avec ces acteurs; nous avons également analysé un volume important d'archives: rapports, courriers, cahiers des charges, dossiers de presse, recommandations stratégiques ${ }^{3}$. Dans la mesure où cet ouvrage a pour objectif de faire le point sur les recherches réalisées ayant trait aux femmes, nous fondons cette contribution sur nos recherches antérieures, mais il n'existe aucune étude actuelle sur le même sujet qui puisse permettre d'actualiser notre investigation. En outre, à cause de la diminution des moyens consacrés à la prévention du sida après 1996, et du fait de l'évolution du profil épidémiologique de l'infection à VIH, l'effort a porté depuis 2000 sur le développement d'une communication ciblée, moins visible mais aussi multiforme et difficile à appréhender dans ce qui n'aurait pu être qu'une mise à jour rapide.

La communication préventive de masse a eu une importance politique majeure. Pendant longtemps, communiquer sur le sida était pour les autorités politiques une preuve de leur souci de la sauvegarde de la santé des citoyens, en évitant des chaussetrapes et écueils opposés : s'immiscer dans la vie privée et faire de la morale, paraître indifférent, manquer de compassion.

Nous nous appuierons ici sur les campagnes de prévention du sida menées de 1987 à 1999 pour montrer comment les figures féminines interviennent et sont prises en compte. Dire que les femmes ont été des laissées pour compte de la communication préventive du VIH serait certainement excessif. Nous voulons surtout montrer que cette cible n'a pas retenu l'attention qu'elle méritait, du fait de l'évolution inexorable de la maladie. II s'agirait donc davantage d'une certaine négligence que d'une véritable maltraitance.

\section{Les prémisses : "Le sida ne passera pas par moi "}

La mise en place de la communication publique sur le sida en France doit être portée au crédit d'une femme, Michèle Barzach, ministre de la Santé en 1987. Son action, unanimement reconnue comme courageuse dans les entretiens que nous avons recueillis, n'a pas concerné seulement la communication. Elle a pris une série de mesures préventives cohérentes: autorisation de la

\footnotetext{
3 Dans le cadre de cette contribution, nous appuierons notre propos surtout sur les archives, documents d'orientation, campagnes de prévention. Le travail d'analyse des campagnes (1987-1996) a été financé par l'Anrs.
}

publicité sur les préservatifs, vente libre de seringues, création des centres de dépistage anonymes et gratuits. Ces mesures constituent une bonne traduction de la réalité épidémiologique du risque : prise en compte des toxicomanes, des multipartenaires, homosexuels ou hétérosexuels, promotion des préservatifs et mise à disposition de l'information sur le statut sérologique.

La communication avait alors pour objectif de faire prendre la mesure de la simplicité et de la sécurité du geste préventif par rapport à la gravité de la menace ${ }^{4}$. Elle devait concerner et impliquer chacun directement ("Devant le sida, nous sommes tous égaux " 5 ), se centrer sur la promotion des préservatifs (" aussi efficaces qu'un vaccin "), bien qu'ils ne soient pas nommés, "rendre le geste de la protection spontané, familier, quasi automatique ", éviter la moralisation.

Le propos central de cette première campagne télévisée était de faire savoir au public - ainsi que l'énonçait une voix off dans le spot télévisé - que le sida n'était " pas une fatalité ", pas une " épidémie " 6 non plus, car l'évocation d'un tel fléau pouvait provoquer des réactions de panique et d'ostracisme, et qu'il « était facile de s'en protéger ". La maladie était concentrée dans des groupes spécifiques et cela était connu de la population. Pour sensibiliser le grand public, il fallait briser cette certitude et convaincre que " tout le monde était concerné par la maladie". Les spots diffusés en avril 1987 montraient un homme ou une femme cassant la courbe inexorablement ascendante du sida. Ils ont été abondamment critiqués par les associations de lutte contre le sida et la presse du fait de leur caractère flou, nulle référence n'étant faite aux préservatifs.

La ministre a intégré ces critiques dans la deuxième vague de la campagne, plus confidentielle car diffusée au cinéma en octobre 1987 : dans la signature, le " moi " cédait la place au " nous ", l'acteur ou l'actrice solitaire était remplacé par un couple, et un préservatif était montré à la fin du spot.

\section{Les femmes : cibles secondaires ou intermédiaires?}

En 1988, les perspectives stratégiques de la communication publique s'affirment et se diversifient mais elles sont objets de controverses. C'est le cas du ciblage : s'adresser à des catégories de population (jeunes ou femmes, notamment) pourrait avoir pour effet pervers de déresponsabiliser les autres (les adultes,

\footnotetext{
C'est ce que préconise un rapport de la SOFRES pour le Comité français d'éducation pour la santé, remis en février 1987. Les citations qui suivent sont extraites de ce rapport.

Une autre version de cette affirmation sera : " le sida concerne tout le monde ", ce qui a pour particularité de gommer la réalité épidémiologique.

Ce terme était soigneusement évité dans la communication publique, de peur de réactions de panique. Nombreux étaient les autorités, les acteurs de la prévention, les membres des associations de lutte contre le sida qui en rejetaient l'emploi, arguant que le sida était une maladie transmissible mais non épidémique, nuance difficile à saisir et abandonnée aujourd'hui. Les termes entre guillemets sont issus du commentaire en voix off des spots télẻvisés.
} 
les hommes). Une campagne télévisuelle de promotion des préservatifs diffusée en novembre 1988 présentait des couples où des femmes tournaient en dérision les résistances des hommes (" II paraît que c'est galère à mettre ") et la signature affirmait en voix off : " Les préservatifs préservent de tout, même du ridicule ». Cette représentation des femmes, assurées, dominatrices, déterminées - personnages phalliques traitant les hommes avec condescendance - va perdurer jusqu'au milieu des années 1990 dans les campagnes de promotion des préservatifs, notamment dans le cadre de spots télévisés. Elle véhicule une image des femmes en décalage avec leur vécu, car elles ont plutôt de la difficulté d'imposer le préservatif à un partenaire qui le refuse [4].

La question de la mise en avant des femmes suscite une divergence entre le ministère de la Santé et le Comité français d'éducation pour la santé (CFES), en charge de la réalisation des campagnes. Le premier souligne dans une note ${ }^{7}$ que les femmes sont une " cible privilégiée ", parce qu'elles sont « courageuses, habituées à être "responsables" de la procréation, de la maîtrise des naissances, (...), de l'unité familiale, de la vie dans son quotidien comme dans sa symbolique (...) Elles sont les « prescripteurs ${ }^{8}$ du préservatif ». Au contraire, le département Études et stratégie du Comité français d'éducation pour la santé affirme, dans un document datant de la même période ${ }^{9}$, que " s'il est vrai que les femmes sont de manière générale plus ouvertes à la prévention, s'il est vrai qu'elles ont pris l'habitude de prendre en charge leur sexualité (contraception, maladies sexuellement transmissibles), s'il est vrai que le sida menace leurs maternités futures et si on peut, pour toutes ces raisons, admettre qu'elles pourront plus facilement changer leurs comportements en ce qui concerne le sida, acheter le préservatif, le proposer, les cibler dans un premier temps de manière spécifique pourrait avoir comme conséquence de déresponsabiliser les hommes ». Dans sa conférence de lancement de la campagne, le ministre de la Santé, Claude Évin, appuie la première option, reprenant dans des termes très proches de la note citée "le rôle primordial de la femme " et leur attribuant une «mission vitale dans la lutte contre la propagation du sida ".

En 1989, alors que la prévention du sida devient la mission d'un organisme spécifique nouvellement créé, l'Agence française de lutte contre le sida, une stratégie est définie et elle perdurera durant toute l'existence de cet organisme, jusqu'en 1994. Elle a un double objectif : promouvoir l'utilisation du préservatif comme un objet de consommation courante, agréable, voire érotique [5], sans référence au sida, et développer un climat de solidarité envers les personnes atteintes. Les femmes y sont considérées comme des cibles secondaires, à moins qu'elles ne fassent partie de la catégorie plus large des "hétérosexuels en période de rencontres, ou ayant plus d'un partenaire par an ». Néanmoins,

* La communication sida *, 14 septembre 1988.

Souligné dans le texte.

* La prévention, bilan et perspectives ", 3 septembre 1988. s'il est « peu souhaitable de leur faire porter tout le poids de la prévention, elles peuvent devenir un vecteur d'influence certain parce qu'elles sont davantage préoccupées par les problèmes de santé ${ }^{10}$. Le fait que les femmes puissent effectivement transmettre le sida dans le cadre de la transmission maternofœtale n'est quasiment jamais abordé ${ }^{11}$ [7]. II est effleuré dans un spot de radio en 1993 et dans un pictogramme adressé aux femmes en grande précarité en 1998. Même si les Antilles ne font pas partie de notre champ d'investigation, nous nous devons de signaler que ce mode de transmission est abordé dans les campagnes de prévention dans les départements français d'Amérique, où les résultats d'une enquête sur la sexualité parue en 1994 mettent bien en évidence, par ailleurs, la vulnérabilité des femmes dans les relations de couple et un double référentiel concernant la sexualité des hommes et des femmes, les uns conquérants et dominateurs et les autres passives et soumises $[7,8]$. En métropole, tout se passe comme si la transmission du VIH de la mère à l'enfant était d'emblée un phénomène honteux concernant les femmes défavorisées ou considérées comme peu éduquées. Ou alors, le silence à son égard démontre une indifférence marquée à des questions qui ne peuvent concerner que les femmes.

Les campagnes de promotion de préservatifs qui se succèdent jusqu'à la fin des années 1990 font bien sûr intervenir des femmes, notamment en tant que membres de couples, mais elles n'évoquent jamais leurs difficultés spécifiques: par exemple, les défaillances dans l'utilisation du préservatif, alors que cette question est traitée dans le cadre de la sexualité des gays. Ces campagnes ne mentionnent pas que le préservatif peut aussi être un contraceptif. Lorsque les femmes sont en couple, elles sont montrées comme maîtrisant bien la question du préservatif dans les relations affectives : pas de préservatif, pas de sexualité et même fin de la relation. C'est ce que martèlent les spots télévisées où l'on voit des femmes se moquer entre elles des hommes qui résistent et renoncer à leur relation (1992), ou des femmes qui claquent la porte au nez d'un homme récalcitrant (1991-1992). D'une certaine façon, montrer ces femmes déterminées, inflexibles, exerce une violence symbolique envers celles qui ont bien plus de mal à se dépêtrer de cette situation conflictuelle. En revanche, les difficultés que rencontrent les femmes face à I'utilisation du préservatif masculin sont bien montrées dans les campagnes du CRIPS, notamment dans certains films de la campagnes « 3000 scénarios pour un virus » en 1994.

Les campagnes de solidarité avec les personnes vivant avec le VIH ont d'emblée mis en scène de façon équilibrée des hommes et des femmes, afin de démontrer que la maladie concernait tout le

\footnotetext{
10 Agence française de lutte contre le sida : « La communication publique sur le sida. Premier document d'orientation $\cdots$, non daté.

11 Les contaminations par ce biais sont restées limitées : entre 1987 et 1996, les cas cumulés de sida issus d'une transmission mère/enfant sont au nombre de 500 environ (BEH $\left.n^{\circ} 38,1996\right)$.
} 
monde ${ }^{12}$. Elles font apparaître, dès la campagne de témoignages de 1989, des femmes séropositives. II est possible de les trouver idéalisées et éloignées des cibles de la maladie. En tout cas, elles étaient montrables, que ce soit dans les spots télévisés ou dans les affiches de la campagne " Dis-moi oui » (1991), par exemple. Ou alors elles étaient mères, compréhensives et aimantes. Parfois jusqu'à l'excès : la campagne « Vivre avec le VIH » de 1995 montrait dans un film un fils vivant avec le VIH et sa mère lovés l'un contre l'autre, dans une relation fusionnelle. Lorsqu'elles sont en couple, les femmes atteintes rencontrent un soutien indéfectible de leur conjoint, comme cela est illustré en 1995, dans le film TV "Le port breton " : le sida permet de vivre son amour et la maladie de façon plus intense, souligne le commentaire. II est à noter que, dans cette campagne de solidarité, deux films présentent des cas de séropositivité dans des couples hétérosexuels où soit la femme, soit l'homme sont atteints, portant ainsi une vision universaliste de la maladie.

Dans la campagne la plus audacieuse jusqu'alors faite sur la prévention sexuelle du sida, durant l'été 1995 , nous retrouvons une volonté similaire de symétrie, cette fois entre des couples hétéro et homosexuels satisfaits de l'utilisation du préservatif. Cependant, dans le cadre du multipartenariat hétéro ou bisexuels, ce sont bien les hommes qui protègent leurs partenaires par cet acte préventif : sous les visuels, le texte s'adresse aux hommes : "Lorsque vous faites l'amour avec Pierre, pensez à protéger Virginie" ou le même texte pour Sophie et Valérie. Comment mieux poser que, par rapport au préservatif, les femmes sont des personnages secondaires?

Cette dernière campagne s'inscrit pourtant dans une nouvelle stratégie qui pouvait laisser présager un traitement différent des femmes. A la dissolution de l'Agence française de lutte contre le sida en 1994, la mise en œuvre de la communication revient au Comité français d'éducation pour la santé et au ministère de la Santé. Sous l'influence des associations de lutte contre le sida et des chercheurs en sciences sociales travaillant sur le sida, la nouvelle stratégie oscille entre rupture et continuité. Des orientations plus complexes se veulent plus proches de l'épidémiologie. II est envisagé de tenir un discours précis et complet à des groupes cibles, définis à partir de typologies prenant en compte le plus de critères possibles, afin, dans l'idéal, d'expliquer à chacun, en fonction de sa situation, les différentes problématiques auxquelles il peut être confronté en matière de prévention du sida. On ne s'intéresse plus uniquement aux risques de transmission sexuelle de la maladie, mais également aux autres modes de contamination (voie sanguine, transmission maternofœtale). Au niveau de la gestion des risques sexuels, il s'agit « de donner toute son importance à l'interaction entre les deux partenaires

\footnotetext{
12 La stratégie de communication définie par l'Agence française de lutte contre le sida à partir de 1989 distingue deux axes de communication donnant lieu à des actions séparées : une communication sur les préservatifs fondée sur un découplage entre préservatifs et sida et une communication sur la proximité aux personnes vivant avec le VIH, ayant pour objectif de maintenir un bon climat de solidarité.
}

dans la gestion d'un risque sexuel, de sorte que la prévention préconisée soit adaptée au style de vie chacun » [2, p. 203]. On hésite donc moins à parler de fidélité et de réduction des risques. Les messages sur le préservatif font explicitement référence au sida, ce qui était banni dans la stratégie précédente fondée sur un découplage entre sida et préservatif.

\section{Prise de conscience sans effet immédiat}

La façon dont les femmes sont représentées ou ciblées n'évolue pas de manière flagrante à la fin des années 1990 alors qu'en même temps, à bas bruit, on observe une inflexion nette du profil de l'épidémie. À partir de 1997, les contaminations hétérosexuelles représentent pourtant une part prédominante des nouveaux cas de sida. Le sex ratio a évolué significativement, passant de une femme pour sept hommes en 1988 à une femme pour trois hommes en $1998^{13}$.

Dans les années 1996-1997, la thématique "femme et VIH " trouve néanmoins progressivement sa place au sein de la prévention du sida. Les partenaires des pouvoirs publics sont plus attentifs à cette question : l'association Aides, pour sa part, introduit, en 1995, une différenciation des genres dans sa communication. Comme cette association, l'administration se dote d'un groupe de travail spécifique en 1996 et établit l'année suivante un état des lieux sur la situation des femmes face au VIH, à partir d'études issues d'organisations telles que le Centre européen pour la surveillance épidémiologique du sida, Aides et Sida info service. Soulignons l'impact des associations de lutte contre le sida pour infléchir les politiques de lutte contre cette maladie, que ce soit pour prendre en compte les revendications des gays ou pour initier une prévention spécifique aux femmes $[9,10]$. Un colloque est organisé au ministère de la Santé sur le thème Femmes et infection à VIH en Europe, en $1997^{14}$. On rappelle à cette occasion que les moyens de protection propres aux femmes ne sont pas encore promus ou disponibles, comme c'est le cas du préservatif féminin, qui sera introduit en France quelques mois plus tard. La question de " l'oubli » des femmes dans les politiques de prévention et dans la communication revient comme un leitmotiv dans la bouche de tous les intervenants, tant des professionnels de la prévention des structures publiques nationales et locales et des acteurs associatifs que des ministres présents: Martine Aubry, Michèle Barzach, Bernard Kouchner et Simone Veil. Cette mobilisation politique, qui va se concrétiser par la suite, va agir comme un déclencheur. Jusqu'alors, les orientations de la politique de prévention du sida et l'évolution

\footnotetext{
13 « Parmi les cas de sida diagnostiqués en 1999, elle [la contamination hétérosexuelle] concerne $41 \%$ des cas, tandis que les homosexuels/bisexuels représentent $29 \%$ des cas et les usagers de drogues injectables $16 \%$. Ces modes de contamination représentaient respectivement $35 \%, 34 \%$ et $19 \%$ du nombre de cas de sida diagnostiqués en 1997 = (BEH, $\left.n^{\circ} 38,2000\right)$.

14 Cf. Femmes et infection à VIH en Europe, 1999, Actes du colloque 14/15 novembre 1997, Paris, CFES, coédité avec le CESES et Sida info service, 1999.
} 
de la situation épidémiologique à travers une prédominance des populations touchées par le sida, via une contamination hétérosexuelle, n'avaient pas véritablement réussi à impulser des changements au niveau de la communication.

Le programme national de réduction des risques envers les femmes, lancé en 1997 par Martine Aubry, ministre de l'Emploi et de la Solidarité, donnera cette impulsion. Le caractère interministériel de la mise en œuvre de ce plan contribue à rapprocher les questions de l'éducation à la sexualité et du sida. Auparavant, l'Agence française de lutte contre le sida avait justifié le fait de passer sous silence l'utilisation du préservatif comme moyen de contraception de la façon suivante : dans l'optique d'une stratégie de séduction publicitaire, la promotion de ce mode de prévention impliquait un message simple dédouané de toute connotation sanitaire. Les militants homosexuels avaient appuyé la séparation du sida et de l'éducation sexuelle pour deux autres raisons. Ils craignaient une banalisation et une "normalisation " de la cause qui devait au contraire conserver son " exceptionnalité ", afin de garder sa place sur l'agenda politique. Cela permettait de plus aux militants associatifs de situer les débats sur un terrain connu (homosexualité et sida) et de conserver ainsi leur position dominante sur d'autres types d'expertises, en particulier sur celles des spécialistes de la santé publique. L'idée de délivrer un message plus globalisant sur la sexualité et les préventions associées ${ }^{15}$ est alors introduite dans les stratégies de communication sur le sida. On décrète également à cette période la nécessité de s'adresser de manière différenciée aux hommes et aux femmes.

Néanmoins, outre la campagne sur la contraception directement élaborée par les services ministériels de Martine Aubry, la mise en œuvre de l'action de communication sur femmes et VIH à la fin des années 1990 connaît des déboires, si bien que ces campagnes ne voient respectivement le jour qu'en 2000 et 2001.

\section{Des actions ciblées et une nouvelle représentation des femmes}

Après 1997, la reconnaissance de la situation spécifique des femmes est censée se traduire par la mise à disposition et la promotion de moyens de contrôle de leur prévention tels que le préservatif féminin. Le manque d'écho dont pâtit ce projet au sein du ministère se trouve renforcé par des doutes sur les possibilités d'une demande à son égard. La distribution des fémidons (préservatifs féminins) semble devoir rester cantonnée à une logique d'offre, en partie en raison du coût de l'objet à l'importation (entre 4 et 5 francs en 1998). Ainsi, entre le moment où Martine Aubry annonce la mise à disposition du préservatif féminin, en 1997, et la première action de communication sur

\footnotetext{
15 CFES, Ed. Programmes de communication et de promotion de la santé sur le sida, le cadre de travail pour les années 2000-2002.
}

le sujet, trois années se sont écoulées. La communication sur le préservatif féminin reste discrète, relayée par des brochures et non par les médias grand public.

Pour de toutes autres raisons, la première campagne de prévention du sida ciblée en direction des femmes lancée en l'an 2000 prend du retard. On le doit à la fois à des ajustements difficiles entre les attentes de la Division sida du ministère de la Santé et les propositions de l'agence de communication. Les premières propositions de créations apparaissent tout à fait inappropriées. C'est notamment le cas d'une affiche avec pour principale représentation le dessin d'un appareil génital féminin dont le but est de faire prendre conscience de la vulnérabilité physiologique des femmes. Des problèmes d'ordre organisationnel au sein du ministère multiplient aussi les étapes de validation et diffèrent sa diffusion.

C'est donc par le biais d'une stratégie ciblant les populations "vulnérables "16 que l'on a communiqué en direction des femmes, dans la mesure où l'on a repéré chez elles deux formes de fragilités face au VIH, physiologique et " socioculturelle ». On souligne un risque beaucoup plus grand que celui des hommes d'être contaminées lors d'une relation sexuelle quand le partenaire est séropositif. Les femmes pâtiraient aussi d'une possibilité réduite de négociation de conduites sans risque lors des relations sexuelles. Cette situation est attribuée à une forme de domination masculine dans ce type d'interaction et aux modes de prévention promus jusqu'alors. Dans le programme de prévention du sida 1997-1999 du ministère de la Santé, il est précisé que " les violences à l'encontre des femmes (violences physiques ou morales), la précarité et certaines formes d'exclusion (prostitution, usage de drogue ...) constituent des facteurs aggravants au regard de la prévention du VIH et des autres maladies sexuellement ${ }^{17}$.

II n'est plus question de vulnérabilité mais de faille dans les comportements de prévention, chez les femmes comme chez les hommes, dans la campagne TV "Confidences" de 1999. Cette action avait pour objectif de faire réfléchir les individus sur les enjeux psychologiques qui sont susceptibles de prendre le pas sur la nécessité de se protéger. Elle raconte des moments dans une relation au cours desquels se nouent des enjeux susceptibles de reléguer au second plan l'utilisation du préservatif. Un film traite de la fragilité de l'après-divorce, thème sur lequel se centre la recherche de Bastard et al. [11], de la peur de briser la confiance dans le cadre d'une rencontre amoureuse entre un homme et une femme d'une quarantaine d'années. Ni l'homme ni la femme n'ont osé aborder la question du préservatif et ils n'en ont pas utilisé. Les raisons invoquées diffèrent. Le premier évoque l'irréalité du risque, le fait de ne pas avoir de

\footnotetext{
16 CFES. Programme de communication et de promotion de la santé sur le sida, le cadre de travail pour les années 2000-2002

17 DGS, Stratégie de prévention de l'infection VIH/sida en France, 2001/2004. Dans les différents plans de communication, cette situation est particulièrement soulignée pour les femmes migrantes et originaires des DOM.
} 
préservatif sous la main, la " peur de ne pas assurer ". Elle avait, en revanche, peur de montrer qu'elle ne lui faisait pas confiance. Un autre film aborde la difficulté pour une personne atteinte de porter seule le poids de la prévention. La jeune femme séropositive est montrée comme exemplaire dans son comportement de prévention, même si elle admet que " ce n'est pas toujours facile de mettre un préservatif $»$.

Au cours des années 2000 , les campagnes sont plutôt marquées par l'ancien modèle de l'indifférenciation des genres, même si une stratégie centrée sur les "populations à risques " se traduit par des messages s'adressant aux femmes appartenant à ces groupes, en l'occurrence aux immigrées et aux jeunes, non plus uniquement dans la communication passant par les médias ciblés, comme c'était le cas auparavant. Parmi les trois films de la campagne de promotion du dépistage lancée à l'occasion du premier décembre 2002, un spot met, pour la première fois, en scène des immigrés dans des messages spécifiques et destinés au grand public. Une femme originaire d'Afrique sub-saharienne demande au téléphone à son compagnon d'avoir recours au test.

Au tournant des années 2000 , nous pouvons prendre la mesure du chemin parcouru au long de près de deux décennies: les problèmes de défaillance sont abordés chez les couples hétérosexuels; la fragilité des femmes face à la prévention par le préservatif est reconnue et la communication permet d'en prendre conscience, de l'aborder, de la gérer, de la dépasser. Les personnes les plus exposées à l'épidémie apparaissent à visage découvert : des femmes migrantes et noires sont montrées dans des spots télévisés. Les pouvoirs publics ont dépassé la crainte de susciter du racisme ou de la mise à distance face à une désignation réaliste qui n'est plus considérée comme de la stigmatisation. Ils ont acquis aussi des savoir-faire par rapport à une communication qui sort des sentiers battus.

\section{Conclusion}

S'interroger sur la façon dont la question des femmes a été abordée dans la communication publique de prévention du VIH revient à poser deux questions complémentaires : comment la cible des femmes, en tant que destinataires spécifiques de la communication, a-t-elle été constituée et s'est modifiée au cours du temps et comment les femmes ont-elles été représentées dans les messages?

Les réponses à ces questions ne sont pas univoques, car l'épidémie d'infection à VIH a évolué au cours du temps depuis la première campagne de prévention en 1987. Elle s'est développée, en débordant les groupes considérés au départ comme les plus exposés. La prise de conscience des vulnérabilités spécifiques des femmes au $\mathrm{VIH}$, notamment des immigrées et des femmes vivant dans les département français d'Outre-Mer, tant physiologiques que sociales, ne s'est faite que très progressivement et ce n'est qu'au tournant des années 2000 qu'une communication ciblée leur est adressée directement. Sur plus de deux décennies, ont évolué aussi les représentations de la sexualité des femmes - mais également celles relatives à la sexualité en général - et de leurs rôles sociaux. Ils ont évolué sans être véritablement bouleversés : les rapports de pouvoir entre hommes et femmes demeurent, en se déplaçant et en perdant un peu de leur intensité.

Depuis 1987, le ciblage des femmes dans la communication a souvent été en porte-à-faux. Elles ont été traitées dans le passé comme cibles intermédiaires. L'accent était alors mis sur leur capacité à relayer l'information car, en tant que gardiennes du foyer, leur rôle social traditionnel en matière de soins et d'éducation était mis en avant. Le risque de transmission de la mère à l'enfant, le risque d'être infectées par leur partenaire régulier, même si elles lui sont fidèles car elles ne peuvent lui imposer le préservatif, les liens entre prévention et contraception, n'ont pratiquement jamais été objets de communication dans les campagnes grand public. Cela illustre les freins à la prise en compte des problèmes spécifiques aux femmes dans le cadre de la communication de prévention du VIH. Celle-ci s'est souvent adressée aux hommes, en montrant des femmes pour bien marquer que le sida était « un problème qui concernait tout le monde $»$.

Les campagnes de promotion du préservatif ont longtemps oscillé entre deux tendances : soit elles supposent que les femmes sont dans une position égalitaire, quand ce n'est pas dominatrice, qui leur donne entière capacité de négocier et cela revient à nier leurs difficultés, voire à les en culpabiliser, soit elles s'adressent à des hommes qui ont des "partenaires " dont le sexe n'a pas besoin d'être spécifié et qui sont « indifféremment » hommes ou femmes.

En revanche, les campagnes de solidarité ont montré une image biaisée des femmes vivant avec le VIH, car idéalisée et, surtout, ne reflétant aucunement la réalité épidémiologique, ni la difficulté de dire l'infection et de reconstruire une vie affective. Au fond, ce n'est que dans les dernières années et sur des cibles spécifiques, dont on a supposé longtemps qu'elles étaient très difficiles à atteindre - femmes des département français d'OutreMer, femmes en précarité, migrantes - et sur des canaux de moins grande diffusion, que la réalité du sida pour les femmes a été abordée, et ce alors que le sex ratio a tendance à s'équilibrer pour les nouvelles infections. Le vécu du risque et de l'infection dans ces populations est bien éloigné des représentations idéalisées qui ont prévalu dans la communication dirigée vers le grand public. On se retrouve cependant dans un dilemme bien connu : la communication ciblée est plus efficace mais elle n'est perceptible que par ses cibles. Peu visible, elle n'informe pas le public de l'engagement des pouvoirs publics, ce qui est souvent perçu comme un inconvénient majeur par ces derniers. En revanche, si elle est visible car diffusée à grande échelle, elle adopte un 
langage plus contraint devant la nécessité de choquer le moins de monde possible, sans qu'on en ait toujours conscience, et ceci la distancie de la réalité.

\section{Références bibliographiques}

1. Löwy I. L'emprise du genre : masculinité, féminité, égalité. Paris : La Dispute, $2005,277 p$.

2. Paicheler G. Prévention du sida et agenda politique: Les campagnes en direction du grand public, Paris : CNRS-Editions, 2002, 284 p.

3. Sitbon A. Dynamiques organisationnelles d'une politique controversée. Une sociologie de la communication publique sur le sida. Thèse de doctorat EHESS, 2006.

4. Paicheler G. et Quemin A. Le public face à la menace du sida. II : Gérer le risque. Paris : Rapport Anrs, 1995.

5. Got C. Rapport sur le sida. Paris : Flammarion, 1989.
6. Couteau M.J. Les campagnes préservatif en France. Journal des Anthropologues 1997 ; 68-69: 57-66.

7. Giraud M. et Gilloire A., Analyse des comportements sexuels aux AntillesGuyanne. Paris : Rapport Anrs, 1994.

8. Le double standard des comportements sexuels aux Antilles et en Guyanne françaises. L'épreuve des faits, la leçon des résultats. In Sexualité, normes et contrôle social. Paicheler G. et Loyola M.A. Paris : L'Harmattan, 2003.

9. Paicheler G. Sauver la vie sexuelle ou imposer des modèles. L'impact des associations sur la communication publique de prévention du sida. In Paicheler G. et Loyola M.A., Sexualité, normes et contrôle social, Paris, L'Harmattan, 2003, 33-52.

10. Paicheler G. Les associations de lutte contre le sida et la communication publique : Une influence minoritaire. Hermès $2005 ; 41$ : 103-110.

11. Bastard B, Cardia-Vonèche L, Peto D. Campenhoudt (Van) L., Relationships between Sexual Partners and Ways of Adapting to the Risk of Aids : Landmarks for a Relationship-oriented Conceptual Framework, In Sexual Interactions and HIV Risk New Conceptual Perspectives in European Research, L Van Campenhoudt, M Cohen, G Guzzardi, D Hauser, Eds. London : Taylor \& Francis, $1997,44-58$. 